\title{
Tuning Electrodeposition Parameters for Tailored Nanoparticle Size, Shape, and Morphology: An In Situ ec-STEM Investigation
}

\author{
Raymond R. Unocic ${ }^{1}$, Robert L. Sacci ${ }^{2}$, Gabriel M. Veith ${ }^{2}$, Nancy J. Dudney ${ }^{2}$, Karren L. More ${ }^{1}$ \\ 1. Center for Nanophase Materials Sciences, Oak Ridge National Laboratory, Oak Ridge, TN 37831 \\ 2. Materials Science Technology Division, Oak Ridge National Laboratory, Oak Ridge, TN 37831
}

Advances in vacuum-tight in situ liquid cell TEM systems have attracted significant attention because of the ability to directly interpret chemical and electrochemical reactions within their native liquid environments [1]. Conducting quantitative in situ electrochemistry experiments within the S/TEM is feasible using the relatively new platform of in situ electrochemical S/TEM (ec-S/TEM). In this approach, microfabricated electrochemical cells are used to seal the liquid electrolyte between two silicon microchips with electron transparent $\mathrm{SiN}_{\mathrm{x}}$ viewing membranes and micropatterned electrodes. The electrochemical microchip devices used in the present study have platinum reference and counter electrodes and a glassy-carbon working electrode (GC-WE) that are directly patterned on the microchip device. Figure 1a shows a schematic of the microfabricated electrochemical cell and vacuum-tight in situ TEM holder assembly. SEM images of the spacer microchip and electrochemical microchip are shown in Figure $1 \mathrm{~b}$ and Figure 1c, respectively. The GC-WE is microfabricated onto the $\operatorname{SiN}_{\mathrm{x}}$ viewing window, which permits the direct visualization of electrochemical reactions occurring directly on the electron transparent glassy-carbon (Figure 1d). Quantitative electrochemical measurements can be performed using these small-scale electrochemical devices via techniques such as cyclic voltammetry (CV), chronoamperometry, and electrochemical impedance spectroscopy, with no adverse effects from the electron beam [2]. In the present study, this method is used to investigate the dynamics of crystal nucleation and growth mechanisms. Moreover, we demonstrate how electrodeposition parameters (CV scan rate) can be adjusted to tailor the size-scale and morphology of electrodeposited nanoparticles.

It has been previously shown that this approach can be use to reduce copper from a $\mathrm{CuSO}_{4}$-based electrolyte onto a gold working electrode, the dynamics of which were captured with TEM imaging [3]. Aberration-corrected HAADF-STEM imaging $\left(\mathrm{C}_{\mathrm{s}}\right.$ corrected FEI Titan S/TEM operating at 300kV), with its increased spatial resolution and the benefits of Z-contrast STEM imaging, was used to characterize the electrodeposited $\mathrm{Cu}$ nanoparticle nucleation and growth events. As a result of the $\mathrm{Cu}$ nanoparticles having a higher atomic number (Z-29), as compared to the glassy-carbon working electrode (Z-6), HAADF-STEM imaging proves to be beneficial because $\mathrm{Cu}$ will have a more intense contrast as compared to the "low Z" glassy carbon background. Figure 2 shows a typical CV from a $0.2 \mathrm{M} \mathrm{CuSO}_{4}$ electrolyte acquired at sweep rates of $50 \mathrm{mV} / \mathrm{s}$ (blue) and $100 \mathrm{mV} / \mathrm{s}$ (red). The $\mathrm{CV}$ data shows several characteristic cathodic and anodic peaks that correlate with $\mathrm{Cu}$ electrodeposition and electrodissolution, respectively. During the CV experiments, HAADF-STEM imaging captured $\mathrm{Cu}$ nucleation on the GC-WE and at the glassy-carbon/ $\mathrm{SiN}_{\mathrm{x}}$ interface. The $\mathrm{CV}$ scan rate had a profound effect on the size scale and distribution of the electrodeposited nanoparticles, with higher scan rates resulting in smaller nanoparticles, as shown in Figures 3a-c and Figure 3d-f for scan rates of $50 \mathrm{mV} / \mathrm{s}$ and $100 \mathrm{mV} / \mathrm{s}$, respectively. The low electron beam current $(0.23 \mathrm{nA})$ and dose rate $\left(6.67 \mathrm{e}^{-} / \mathrm{nm}^{2}-\mathrm{s}\right)$ used in these experiments is below the threshold for $\mathrm{Cu}$ reduction by the electron beam, which is clearly evident by the lack of $\mathrm{Cu}$ nanoparticles on the $\mathrm{SiN}_{\mathrm{x}}$ viewing window adjacent to the edge of the GC-WE interface. It is expected that under higher electron beam currents and higher electron dose rates, $\mathrm{Cu}$ can 
readily be reduced on the $\mathrm{SiN}_{\mathrm{x}}$ membranes from the $\mathrm{CuSO}_{4}$ electrolyte [4]. This work provides the framework for directly interpreting the fundamental mechanisms of crystal nucleation and growth [5].

\section{References:}

[1] N. de Jonge and F. M. Ross, Nature Nanotechnology, 6, (2011), 695-704.

[2] R.R. Unocic, et al., Microscopy and Microanalysis, (2014), in press.

[3] M.J. Williamson, et al., Nature Materials, 2, (2003), 532-536.

[4] T.J. Woehl, et al., Ultramicroscopy, 127, (2013), 53-63.

[5] Research supported by Oak Ridge National Laboratory's Center for Nanophase Materials Sciences (CNMS), which is sponsored by the Scientific User Facilities Division, Office of Basic Energy

Sciences, U.S. Department of Energy.

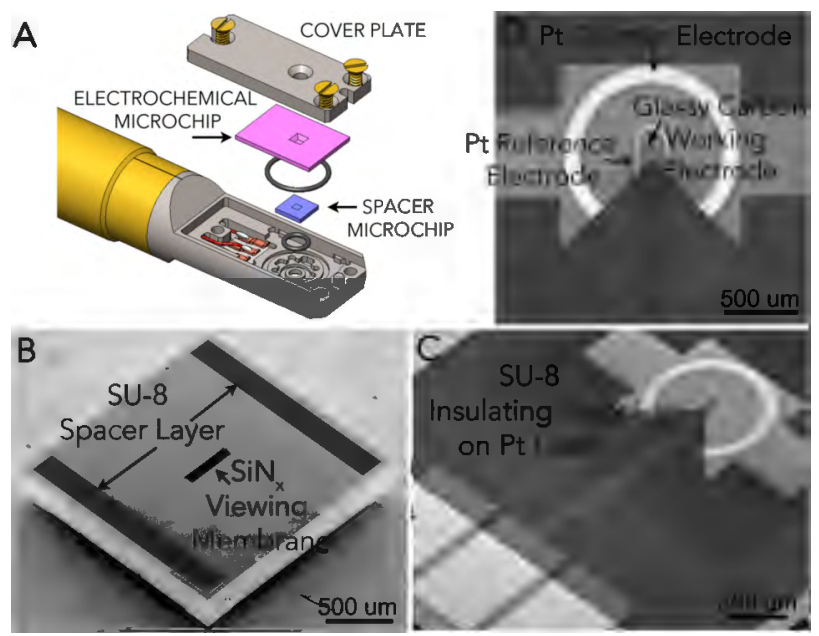

Figure 1. a) Schematic of the in situ ec-S/TEM system. SEM images of the b) spacer microchip and c-d) electrochemical microchip [2].

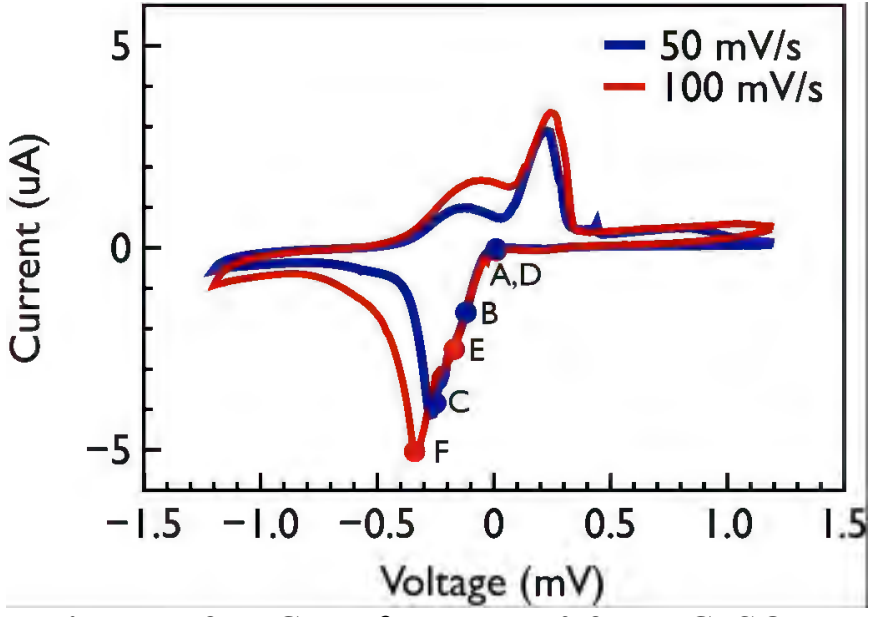

Figure 2. $\mathrm{CV}$ from a $0.2 \mathrm{M} \quad \mathrm{CuSO}_{4}$ electrolyte acquired at scan rates of $50 \mathrm{mV} / \mathrm{s}$ (blue) and $100 \mathrm{mV} / \mathrm{s}$ scan rate (red).

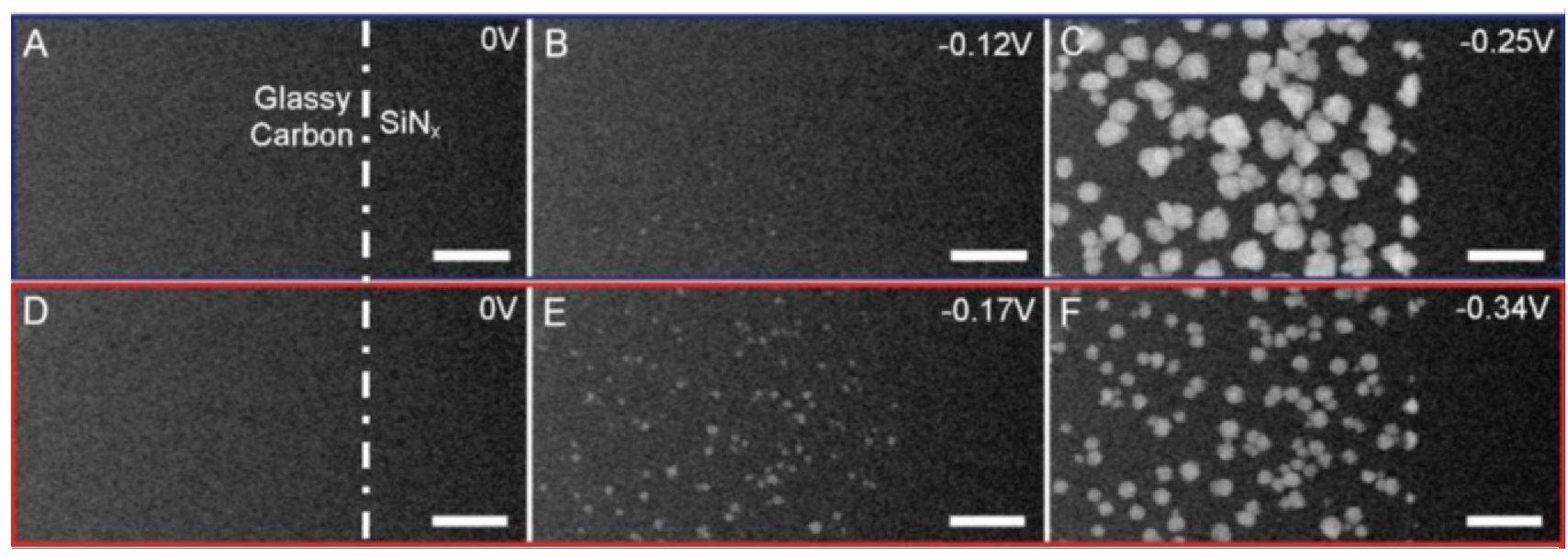

Figure 3. Series of HAADF-STEM images showing the electrodeposition of $\mathrm{Cu}$ on a glassy-carbon working electrode under different CV scan rates: a-c) $50 \mathrm{mV} / \mathrm{s}$ and d-f) and $100 \mathrm{mV} / \mathrm{s}$. Scale bar is $2 \mu \mathrm{m}$. 